\title{
ASSOCIATION OF PLACENTA PREVIA WITH A HISTORY OF PREVIOUS CESARIAN DELIVERIES AND INDICATIONS FOR A POSSIBLE ROLE OF A GENETIC COMPONENT
}

\author{
Matalliotakis $\mathrm{M}^{1, *}$, Velegrakis $\mathrm{A}^{1}$, Goulielmos $\mathrm{GN}^{2}$, Niraki E ${ }^{1}$, Patelarou $\mathrm{AE}^{3}$, Matalliotakis $\mathrm{I}^{1}$
}

\begin{abstract}
*Corresponding Author: Dr. Michail Matalliotakis, Venizeleio \& Pananio General Hospital of Heraklion, Knossos Avenue, 71409 Heraklion, Greece. Tel: +30-281-036-8304. Mobile: +30-694-386-1582. Fax: +30-281-036-8305. E-mail: mihalismat@hotmail.com
\end{abstract}

\begin{abstract}
A prior Cesaria section (C-section) is an important risk factor that leads to endometrial damage and abnormal implantation of the placenta. Our retrospective study aims to correlate the frequency of placenta previa to previous $\mathrm{C}$-sections, to determine the effect of male gender in this condition and to evaluate further the maternal outcome. Seventy-six cases with placenta previa were selected out of 5200 live births. Diagnosis was confirmed by ultrasound and in the operating theater. In the 76 women examined, we found 50 cases with a history of a previous $\mathrm{C}$-section $(66.0 \%)$ and 49 male offspring $(65.0 \%)(p<0.001)$, with a mean birth weight of $2635 \pm 740 \mathrm{~g}$. Of all these patients, six $(8.0 \%)$ cases developed placenta percreta, seven $(9.0 \%)$ were transferred to the intensive care unit (ICU), 14 (18.0\%) women needed blood transfusion and eight (11.0\%) underwent hysterectomy. The results of our series show a strong correlation of placenta previa to a history of previous $\mathrm{C}$-sections and a predominance of male fetuses. Early recognition and proper monitoring could minimize the possibility of a poor outcome.

Keywords: Cesarian section (C-section); Genetics; Male gender domination; Placenta previa.
\end{abstract}

\section{INTRODUCTION}

Placenta previa is a serious obstetric complication of pregnancy, representing a major cause of vaginal bleeding

\footnotetext{
${ }^{1}$ Department of Obstetrics and Gynecology, Venizeleio General Hospital, Heraklion, Greece

${ }^{2}$ Section of Molecular Pathology and Human Genetics, Department of Internal Medicine, School of Medicine, University of Crete, Heraklion, Crete

${ }^{3}$ Department of Nursing, Technological and Educational Institute of Crete
}

after 20 weeks of gestation in which the placenta is partially or fully attached to the internal cervical ostium (os) and may develop into adverse maternal and fetal outcome [1]. The frequency among all pregnancies is approximately $0.3-0.5 \%$, with predominant risk factors being advanced maternal age, gestation with male fetuses, smoking, multiparity, history of previous Cesarian sections (C-sections) and/or habitual abortion, conditions that may lead to damage of the endometrial tissue [2]. The etiology remains enigmatic but some risk factors seem to be superior.

According to the trophotropic theory, the placenta migrates to better vascularized areas. Normally, the placenta grows towards the fundus, which can provide more blood. Defective vascularization of the endometrium due to scarring or atrophy caused by previous operations or infections may result in reduced differential growth of the lower uterine segment and less of an upward shift in placental location [3].

There is an evident literature support that male gender correlates with the low implantation of the placenta. MacGillivray et al. [4] proposed that the time of insemination during the menstrual cycle plays a role in male conception and the site of the implantation. It has been previously suggested that a multi disciplinary medical team is needed for further evaluation and management of these cases because a massive hemorrhage may need obstetric hysterectomy, intensive care and blood transfusions [5]. In the framework of this study, we sought to correlate the frequency of placenta previa to previous $\mathrm{C}$-sections, to determine the effect of male gender in this condition and to further evaluate the maternal outcome.

\section{MATERIALS AND METHODS}

This study was conducted at the Obstetric and Gynecology Department, Venizeleio General Hospital, Her- 
aklion, Crete, and involved all cases between 2010 and 2016; written approval was obtained from all the patients. Of the 5200 deliveries, 76 women with placenta previa were included and divided into two main groups, women with or without a previous history of C-sections. Placenta mapping was done antenatally on transabdominal ultrasound and confirmed in the operating theatre. All operative procedures were entered in the operative book.

A detailed history including the age, the history of previous C-sections, parity and episodes of bleeding during the pregnancy were noted. In our inclusion criteria, we selected 75 singleton pregnancies and one twin (dichorionic diamniotic) pregnancy. The primary exclusion criteria were the marginal type of placenta and the cases where medical notes were unavailable or incomplete. Additionally, all patients were evaluated for placenta acreta or percreta preoperatively. All women delivered before 37 weeks of gestation. Furthermore, based on the review of the medical database, we evaluate the coincidence of placenta previa and male fetus.

\section{RESULTS}

In total, 76 cases were recruited in the framework of this study. The age distribution showed that 26 women (34.0\%) were between 26-30 years old, 23 (30.0\%) between 36-40 years old, 17 (22.4\%) between 31-35, and 10 (13.0\%) cases between 21-25 years old.

The incidence of placenta previa was higher in patients with prior $\mathrm{C}$-sections. We found that 50 women $(66.0 \%)$ had a history of previous C-sections. The percentage with one prior $\mathrm{C}$-section was $46.0 \%$ (23 cases) and $54.0 \%$ (27 cases) with two or more prior C-sections. Of all these patients, six $(8.0 \%)$ cases developed placenta percreta, seven $(9.0 \%)$ were transferred to the intensive care unit (ICU), 14 (18.0\%) women needed blood transfusions, and eight (11.0\%) underwent a hysterectomy (Figure 1).

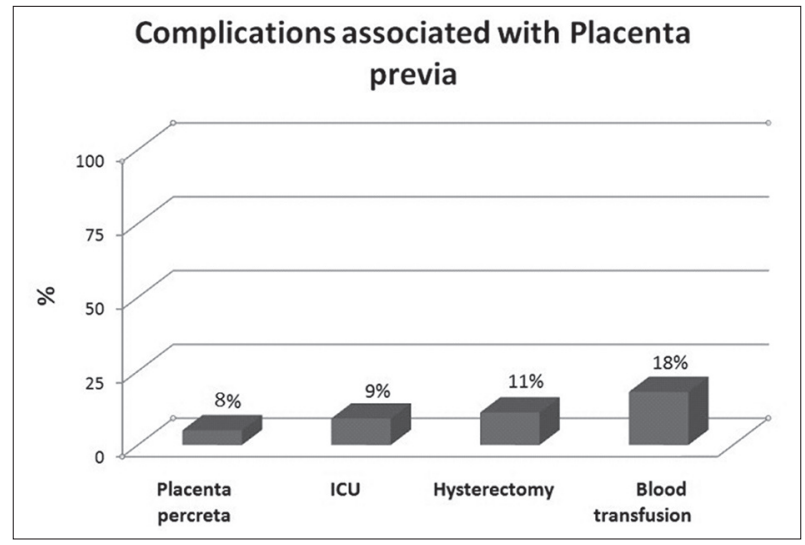

Figure 1. Complications associated with placenta previa.
As far as the offspring was concerned, the data suggested a slightly greater female to male ratio $(51.0 / 49.0 \%)$ in the general population between 2010 and 2016. In our study, the male to female ratio was significantly higher in placenta previa cases. We reported 50 males $(66.0 \%)$ out of 76 live births.

According to the results of the present study, a woman gave birth to three children and placenta previa was observed in all cases. Moreover, three out of six women with placenta percreta were related to each other.

\section{DISCUSSION}

In the current study, we investigated pregnancies associated with a placental dysfunction, placenta previa, in the genetically and environmentally homogeneous population of Crete, Greece. Placenta previa represents the placenta that partially or wholly covers the internal cervical os. Our data indicate that the frequency of observed placenta previa is slightly higher than the one reported so far, which ranges between 0.3 and $0.5 \%$ [6,7].Thus, 76 cases out of 5200 live births had placenta previa (1.46\%). As a possible explanation, this inconsistency may be considered to be due to the very low number of specialized tertiary centers that are available for a large population.

Placenta previa is subdivided into three main types that can be confirmed by a transvaginal scan. Complete form means that the placenta totally covers the cervical os, partial that the edge of the placenta partially covers the birth canal and marginal indicates that the placenta lies close to the cervical os [8].

Several studies suggested that a previous history of $\mathrm{C}$-section is the primary risk factor for developing placenta previa. Data show up to $37.5 \%$ increased risk due to previous history $[9,10]$. In the present study, 50 out of 76 women $(66.0 \%)$ had a history of previous C-sections, a situation that can be explained by the fact that the endometrial cells located close to the scar are unable to differentiate properly resulting in a defective implantation mechanism. Interestingly, it has been reported that the rate of placenta previa increases proportionally with the history of previous C-sections, which is also consistent in our case [11].

Placenta percreta is a potentially lethal complication. It is a result of failure of decidua basalis after a repair of a previous uterine scar, and as a consequence, the chorionic villi invade the myometrium. Hudon et al. [12] stated that women who have both placenta previa and previous uterine scarring are susceptible to develop placenta acreta. In a total of 76 patients with placenta previa, six of them developed placenta percreta. In this group, all women were 
older than 36 and had a history of more than one previous C-section. According to the literature, placenta previa is more common among increased age group ( $>30$ years old), which is in line with the data of the present study. Although, there is not any statistically difference between ultrasound and magnetic resonance imaging (MRI) for diagnosing placenta percreta, high risk cases should be carefully evaluated by MRI in order to determine the degree of the invasion to adjacent organs. It was suggested in the literature to use MRI imaging when the placenta is located in the posterior wall of the uterus $[13,14]$. In the evaluation of the related complications in our series, we reported that seven cases were transferred to the ICU, 14 cases needed blood transfusions and eight underwent hysterectomy due to placenta invasion into the bladder, which was confirmed by MRI. As far as the time of delivery is concerned, each case should be individualized depending on the patient's preferences and risks. A planned preterm hysterectomy after 34 weeks where fetal lungs have matured is recommended, with the placenta being left in situ, to avoid massive bleeding in cases where invasion of the chorionic villi is prominent. However, if the mother has a desire for future fertilization, surgical management should be individualized [15].

Upon now, a few studies have linked the male gender with placenta previa formation. The gender at birth seems to be influenced by variable factors such as parental age, race, psychological status, exposure to hormonal treatment for subfertility and the time of the insemination within the menstrual cycle. The quality of these series is limited because of the incompleteness of the information presented in the reports and the small number of cases [16]. Notably, Jakobovits and Zubek [17] reported an association between male gender and placenta previa at the extreme ends of maternal age. Moreover, it has been proposed that early and late insemination during the menstrual cycle may contribute to male conceptions and also leads to a change in the site of implantation [4].

It is postulated that a male fetus is more likely to develop from late fertilization during the cycle, as a result in a delay in the creation and implantation of blastocyst in the lower uterine segment. Our results suggest that, our results suggest that male gender dominance has become obvious. Out of 76 cases, we reported 49 (65.0\%) male newborns. In a population based study in Canada, 433,031 pregnancy outcomes were collected. The male-to-female ratio was higher in cases complicated by a placenta previa than in those without [18]. It has been suggested that the male embryo is a possible risk factor that we should take into consideration, especially in cases with a history of previous $\mathrm{C}$-sections that may be associated with invasion of the placenta further to other organs.

Although the risk factors for placenta previa are well defined, much less is known about its etiology and the possible enrolment of genetic factors in the development of this condition. In the present study, a woman gave birth to three children and placenta previa was observed in all cases. Moreover, three out of six women with placenta percreta were related to each other. Altogether, these observations indicate that both conditions could be genetically influenced, although no clear genetic contributions to susceptibility have thus far been defined. Placenta previa has an overall prevalence in North America of 2.9/1000 pregnancies, compared with a global prevalence of 5.2/ 1000 pregnancies [19], while the highest prevalence internationally was found in Asian women, appearing an overall prevalence is $12.2 / 1000$ pregnancies. These regional differences in these rates have not been explained in depth, although Iyasu et al. [20] reported that in the United States, women of Asian ethnicity had twice the risk of placenta previa compared with women of other ethnicities. This latter finding strengthens the role of genetics rather than the effect of environmental factors as regards the development of placenta previa in Asian women.

Interestingly, the role of genetics in placenta previa has now been demonstrated. Studies aiming to identify differentially expressed genes, which may impair placentation resulting in placenta previa increta/percreta $(\mathrm{I} / \mathrm{P})$, have shown that the metastasis associated lung adenocarcinoma transcript 1 (MALAT-1) (also known as HCN, NEAT2 and PRO2853) gene expression in placenta previa $\mathrm{I} / \mathrm{P}$ is increased and its down-regulation inhibits trophoblast-like cell invasion in vitro. Placenta previa increta/percreta, a severe form of invasive placentation (a collective term for placenta accreta, increta and percreta), is associated with potentially life-threatening hemorrhage, which often requires a Cesarean hysterectomy. Therefore, it has been assumed that MALAT-1 might be involved in regulating trophoblast invasion during the development of advanced invasive placentation [21]. Additionally, the role of epigenetics in pregnancies associated with placental dysfunction including placenta previa has been also pointed out, indicating that the contribution of epigenetic alterations can be detected in susceptible subjects before the onset of clinical disease. In particular, the involvement of "hypermethylation" has also been reported in placenta previa. Thus, it has been demonstrated that an increased concentration of fetal-derived hypermethylated sequences of RASSF1A (Ras association domain-containing protein 1) tumor suppressor gene according to advancing gestation 
and before the onset of placenta previa [22]. Additionally, and contrarily to placenta previa, a remarkable genetic predisposition has been reported for other placentationassociated obstetric complications such as placental abruption and postpartum hemorrhage $[6,23]$.

\section{CONCLUSIONS}

All women over 30 years old, with one or more previous C-sections, anterior placenta and male fetus should be carefully monitored prenatally, as they may have a higher chance to aquire placenta previa and placenta acreta or percreta. It is necessary to consult the pregnant woman as soon as the pregnancy is confirmed. Early recognition and proper monitoring could minimize the possibility of a poor outcome. Considering a part of the findings of the current study, which are reminiscent of an underlying genetic basis of placenta previa, future research is required to elucidate a possible genetic and/or environmental contribution to risk.

\section{ACKNOWLEDGMENTS}

We would like to thank all the practitioners for providing the pathology reports.

Declaration of Interest. The authors report no conflicts of interest. The authors alone are responsible for the content and writing of this article.

\section{REFERENCES}

1. Kumaran A, Warren R, Sabaratnam. Best Practice in Labour and Delivery, 1st ed. Cambridge, Cambridgeshire, UK: Cambridge University Press. 2009: 142-146.

2. Oyelese Y, Smulian JC. Placenta previa, placenta accreta, and vasa previa. Obstet Gynecol. 2006; 107(4): 927-941.

3. Dashe JS, McIntire DD, Ramus RM, Santos-Ramos R, Twickler DM. Persistence of placenta previa according to gestational age at ultrasound detection. Obstet Gynecol. 2002; 99(5 Pt 1):692-697.

4. MacGillivray I, Davey D, Isaacs S. Placenta praevia and sex ratio at birth. Br Med J (Clin Res Ed) 1986; 292(6517): 371-372.

5. Chou MM, HoEs,Lee YH. Prenatal diagnosis of placenta previa accreta by transabdomilnal color Doppler ultrasound: Ultrasound Obstet Gynecol 2000;15(1):28-35.
6. Ananth CV, Peltier MR, Chavez MR, Kirby RS, Getahun D, Vintzileos AM. Recurrence of ischemic placental disease. Obstet Gynecol. 2007; 110(1): 128-133.

7. Baskett TF, Calder AA, Arulkumaran S. Ante-partum haemorrhage. Munro Kerr's Operative Obstetrics, 12 ed. Toronto, ON, Canada: Elsevier. 2014: 178-200.

8. Oppenheimer W L, Farin D, Ritchie K, Lovinsky RM, Telford J, Fairbanks LA. What is a low-lying placenta? Am J Obstet Gynecol. 1991; 165(4 Pt 1): 1036-1038.

9. Getahun D, Oyelese Y, Salihu HM, Ananth CV. Previous Cesarean Delivery and Risks of Placenta Previa and Placental Abruption. Obstetrics \& Gynecology 2006; 107(4): 771-778.

10. Garmi G, Salim R. Epidemiology, etiology, diagnosis, and management of placenta accreta. Obstet Gynecol Int. 2012; 2012: 873929.

11. Usta IM, Hobeika EM, Musa AA, Gabriel GE, Nassar AH. Placenta previa-accreta: Risk factors and complications. Am J Obstet Gynecol. 2005; 193(3): 1045-1049.

12. Hudon L, Belfort MA, Broome DR. Diagnosis and management of placenta percreta: A review. Obstet Gynecol Surv. 1998; 53(8): 509-517.

13. Masselli G, Brunelli R, Casciani E, Polettini E, Piccioni MG, Anceschi M, et al. Magnetic resonance imaging in the evaluation of placental adhesive disorders: Correlation with color Doppler ultrasound. Eur Radiol. 2008; 18(6): 1292-1299.

14. Varghese B, Singh N, George RA, Gilvaz S. Magnetic resonance imaging of placenta accrete. Indian J Radiol Imaging. 2013; 23(4): 379-385.

15. Belfort MA, Publications Committee, Society for Maternal-Fetal Medicine. Placenta accreta. Am J Obstet Gynecol. 2010; 203(5): 430-439.

16. James WH. The human sex ratio. Part 1: A review of the literature. Hum Biol. 1987; 59(5): 721-752.

17. Jakobovits AA, Zubek L.Sex ratio and placenta praevia. Acta Obstet Gynecol Scand. 1989; 68(6): 503-505.

18. Wen SW, Demissie K, Liu S, Marcoux S, Kramer MS. Placenta praevia and male sex at birth: Results from a population-based study. Paediatr Perinat Epidemiol. 2000; 14(4): 300-304.

19. Cresswell JA, Ronsmans C, Calvert C, Filippi V. Prevalence of placenta praevia by world region: A systematic review and meta-analysis. Trop Med Int Health. 2013; 18(6): 712-724. 
20. Iyasu S, Saftlas AK, Rowley DL, Koonin LM, Lawson HW, Atrash HK. The epidemiology of placenta previa in the United States, 1979 through 1987. Am J Obstet Gynecol. 1993; 168(5): 1424-1429.

21. Tseng JJ, Hsieh YT, Hsu SL, Chou MM. Metastasis associated lung adenocarcinoma transcript 1 is up-regulated in placenta previa increta/percreta and strongly associated with trophoblast-like cell invasion in vitro. Mol Hum Reprod. 2009; 15(11): 725-731.
22. Kim MJ, Kim SY, Park SY, Ahn HK, Chung JH, Ryu HM. Association of fetal-derived hypermethylated RASSF1A concentration in placenta mediated pregnancy complications. Placenta. 2013; 34(1): 57-61.

23. Oberg AS, Hernandéz-Diaź S, Frisell T, Greene MF, Almqvist C, Bateman BT. Genetic contribution to postpartum haemorrhage in Swedish population: Cohort study of 466,686 births. BMJ. 2014; 349: g4984. doi: 10.1136/bmj. g4984. 